\title{
Guest editorial: Special issue on robot communication challenges: real-world problems, systems, and methods
}

\author{
Michael Otte ${ }^{1} \cdot$ Donald Sofge $^{3} \cdot$ Robert Fitch $^{2}$
}

Published online: 3 January 2020

(c) Springer Science+Business Media, LLC, part of Springer Nature 2020

Robots and multi-robot teams use communication to facilitate data sharing, coordination, and cooperation with other robots and human users. Real-world communication is often unreliable, expensive, non-ideal, and/or otherwise challenging in a variety of ways. These challenges lead to interesting theoretical and practical ramifications for the design, analysis, and deployment of robotic systems, algorithms, and hardware. This special issue presents state-of-the art research at the intersection of communication and robotics.

Submissions to this special issue were solicited in an open call. Out of 12 initial submissions, 6 papers were eventually accepted for publication after a rigorous peer review process (an acceptance rate of 50\%). The six articles appearing in this issue span a variety of topics at the convergence of robotics and communication. These topics include: communication challenges faced by robots in underwater environments, the analysis of how limited and/or unreliable communication affects the performance of multi-robot algorithms, the presentation of a number of new multi-robot algorithms designed specifically for cases of constrained communication, and a new communication protocol designed for swarms that communicate over the infrared medium. A highlevel summary of each of the six accepted articles appears below.

The special issue begins with "Transmission of images by unmanned underwater vehicles." This paper, by Alice Danckaers and Mae L. Seto, presents a method for transmission

Michael Otte

otte@umd.edu

Donald Sofge

donald.sofge@nrl.navy.mil

Robert Fitch

robert.fitch@uts.edu.au

1 University of Maryland, College Park, USA

2 University of Technology Sydney, Sydney, Australia

3 U.S. Naval Research Laboratory, Washington, USA of image data via an acoustic communication channel used in the challenging underwater domain. A significant contribution of this work is the experimental validation of the method using indoor water tanks and full-scale experiments at sea.

The next article, titled "Statistics of the distance traveled until successful connectivity for unmanned vehicles," by Arjun Muralidharan and Yasamin Mostofi, presents an indepth statistical analysis of the distance a robot must travel along a path before establishing communication connectivity. This theoretical analysis accounts for path loss, shadowing, and multi-path effects. Validation of the analysis is performed using communication channel parameters from downtown San Francisco.

To improve multi-robot collaborative data sharing over a communication medium with limited bandwidth, an algorithm for quickly estimating the collaborative utility of a message is presented in "Optimizing multi-robot communication under bandwidth constraints" by Ryan J. Marcotte, Xipeng Wang, Dhanvin Mehta, Edwin Olson. The method is evaluated within a multi-robot navigation scenario.

Two new methods for tracking multiple targets using multiple agents with limited communication are presented in "Distributed assignment with limited communication for multi-robot multi-target tracking" by Yoonchang Sung, Ashish Kumar Budhiraja, Ryan K. Williams, Pratap Tokekar. Both of the algorithms presented in this paper have bounded suboptimality, where the bounds are given in terms of the number of communication rounds that are permitted.

A multi-cast algorithm designed for scenarios where both the source and the receiver nodes are mobile appears in "Live multi-cast video streaming from drones: an experimental study" by Raheeb Muzaffar, Evşen Yanmaz, Christian Raffelsberger, Andrea Cavallaro. The algorithm is experimentally validated by streaming video over an aerial ad-hoc IEEE 802.11 network.

The final paper of the issue is titled "SwarmCom: an infra-red-based mobile ad-hoc network for severely 
constrained robots," and is by Stefan M. Trenkwalder, Iñaki Esnaola, Yuri Kaszubowski Lopes, Andreas KollingRoderich Groß. This paper presents a mobile ad-hoc network protocol for swarm communication that is designed for infrared optical channels. The new protocol is compared to existing approaches on swarms of e-puck robots.

The idea for a special issue at the intersection of robotics and communication grew out of the success of the workshop "Robot Communication in the Wild: Meeting the Challenges of Real-World Systems" that was held as part of the Robots Science and Systems Conference (RSS) in 2017. This workshop was organized by Robert Fitch, Donald Sofge, Geoffrey Hollinger, Karthik Dantu, Michael Otte, and Shayegan Omidshafiei. All articles appearing in the current special issue were submitted in response to an open call and have been rigorously peer reviewed according to the high standards of Autonomous Robots.
The guest editors gratefully acknowledge the scientific contributions of the authors and the critically important work of the peer reviewers. The Springer editorial staff has been a pleasure to work with and was integral to making this special issue a success. We would also like to thank Editor-in-Chief Gaurav Sukhatme for supporting a special issue on this topic.

Publisher's Note Springer Nature remains neutral with regard to jurisdictional claims in published maps and institutional affiliations. 